\title{
Symmetry of Coherent Vortices in Plane Couette Flow
}

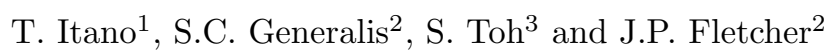

${ }^{1}$ Faculty of Engineering Science, Kansai University, 564-8680 Osaka, Japan

itano at kansai-u.ac.jp

${ }^{2}$ School of Engineering \& Applied Sciences, Aston University, B4 7ET

Birmingham, UK.

${ }^{3}$ Department of Physics and Astronomy, Graduate School of Science, Kyoto

University, Kyoto 606-8502, Japan

A numerical continuation method is carried out in a homotopy space connecting two different flows, the Plane Couette Flow (PCF) and the Laterally Heated Flow in a vertical slot (LHF). This numerical continuation method enables us to obtain an exact steady solution in PCF [6]. The new solution has the shape of hairpin vortices (HVS: hairpin vortex solution, see Fig.1), which is observed ubiquitously in turbulent shear flows.

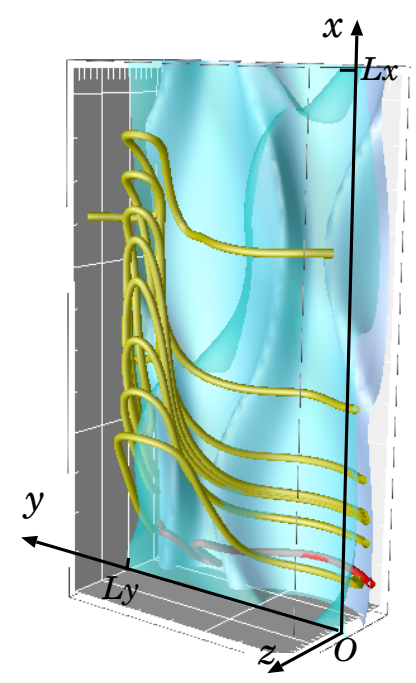

Fig. 1. The hairpin vortex solution (HVS) in plane Couette flow (PCF) at $R e=200$. (Yellow) curves are vortex lines across the channel mid-plane, underneath which there are low-speed structures visualised as (cyan) isosurfaces. Here $x, y, z$ denote the stream-, span-wise and wall coordinates. 
The HVS satisfies three distinct symmetries, which lead to the apparent spanwise reflection symmetry across a plane $\left(y=L_{y} / 4\right.$ or $\left.3 L_{y} / 4\right)$ as seen in Fig.1. By contrast, only two of the three symmetries are satisfied by the streamwise vortex solution (SVS), which was previously obtained as a coherent structure typical of wall turbulence in Refs.[1, 2, 3, 4]. Thus, the SVS is expected to bifurcate from the HVS via breaking of the spanwise reflection symmetry depicted in Fig.1. In the present study, we show evidence that the SVS bifurcates from the HVS in the homotopy space between the PCF and the LHF.

We first consider an incompressible Boussinesq fluid with $\operatorname{Pr}=0$ filling a vertical slot of thickness $2 \tilde{h}$. The boundaries of the gap are two rigid parallel planes of infinite extent heated laterally with temperatures $\tilde{T}_{0} \pm \Delta \tilde{T}$, and which move relative to each other with speed $2 \Delta \tilde{U}$ in the x-direction of the Cartesian coordinate system. In terms of the two non-dimensional parameters, $R e=$ $(\tilde{A}+\Delta \tilde{U}) \tilde{h} / \tilde{\nu}$ and $\epsilon=\tilde{A} /(\tilde{A}+\Delta \tilde{U})$, where $\tilde{A}=\tilde{\gamma} \tilde{g} \Delta \tilde{T} \tilde{h}^{2} / 6 \tilde{\nu}$, the perturbation from the static state of our system is governed by the incompressibility and momentum equations respectively:

$$
\boldsymbol{\nabla} \cdot \boldsymbol{u}=0, \quad \boldsymbol{u} \cdot \boldsymbol{\nabla} \boldsymbol{u}=-\nabla p+\frac{6 \epsilon}{R e} z \boldsymbol{e}_{x}+\frac{1}{R e} \nabla^{2} \boldsymbol{u} .
$$

Here, the parameter $\epsilon$ plays an important role in our analysis; solutions obtained with $\epsilon=0$ are exact states of PCF, while those with $\epsilon=1$ are exact solutions of LHF.

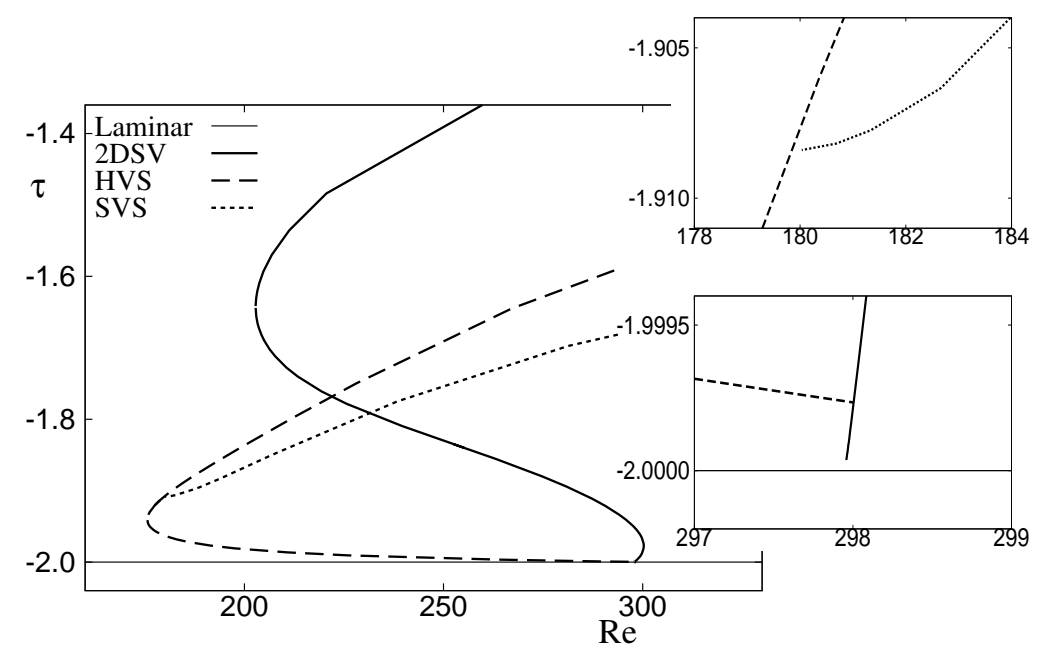

Fig. 2. Transition in $\operatorname{LHF}(\epsilon=1):(\alpha, \beta)=(2.0,0)$ for the $2 \mathrm{DSV}$ (2D spanwise vortex), $(\alpha, \beta)=(1.0,2.0)$ for the tertiary state (abbreviated as "HVS") and the SVS. 
Fig.2 shows the bifurcation diagram obtained in LHF $(\epsilon=1)$, where the mean shear rate at the boundary, $\tau$, is adopted as an order parameter to characterise solutions. The secondary branch in LHF $(\epsilon=1)$ that bifurcates from the laminar state in LHF (see the right-bottom inset of Fig.2) consists of the two-dimensional spanwise vortical (2DSV) structures, which exhibit cat's-eye roll patterns occupying the whole channel width (cf. Ref.[5]). The branch satisfies the following symmetries: "Spanwise translational symmetry" $\boldsymbol{u}(x, y, z)=\boldsymbol{u}(x, y+\Delta y, z)$, and "Rotational symmetry with respect to the $\mathrm{y}$ axis" $\left[u_{x}, u_{z}\right]^{T}(x, y, z)=\left[-u_{x},-u_{z}\right]^{T}(-x, y,-z)$.

The tertiary branch that bifurcates from 2DSV, termed as "HVS" in the diagram, is the new haipin vortex solution reported recently in [6]. The branch satisfies the following three independent symmetries:

$\mathcal{A}$ "Streamwise translational and spanwise reflectional symmetry", $\left[u_{x}, u_{y}, u_{z}\right]^{T}(x, y, z)=\left[u_{x},-u_{y}, u_{z}\right]^{T}\left(x+L_{x} / 2,-y, z\right)$,

$\mathcal{B}$ "Parity symmetry with respect to $(x, y, z)=\left(L_{x} / 4, L_{y} / 4,0\right)$ ", $\left[u_{x}, u_{y}, u_{z}\right]^{T}\left(\frac{L_{x}}{4}+x, \frac{L_{y}}{4}+y, z\right)=\left[-u_{x},-u_{y},-u_{z}\right]^{T}\left(\frac{L_{x}}{4}-x, \frac{L_{y}}{4}-y,-z\right)$,

$\mathcal{C}$ "Parity symmetry with respect to the origin", $\left[u_{x}, u_{y}, u_{z}\right]^{T}(x, y, z)=\left[-u_{x},-u_{y},-u_{z}\right]^{T}(-x,-y,-z)$,

where $L_{x}$ and $L_{y}$ are the streamwise and spanwise wave-lengths of the HVS. The spanwise reflection symmetry of HVS, $\left[u_{x}, u_{y}, u_{z}\right]^{T}\left(x, L_{y} / 4+y, z\right)=$ $\left[u_{x}, u_{y}, u_{z}\right]^{T}\left(x, L_{y} / 4-y, z\right)$, can be deduced by the symmetries $\mathcal{A}, \mathcal{B}$ and $\mathcal{C}$. By employing the numerical continuation method, this branch connects to a solution at the PCF limit $(\epsilon=0)$, which contains a hairpin-shaped bundle of vortex lines depicted in Fig.1.

Furthermore, a branch (termed as "SVS" in the diagram) bifurcates from HVS at $R e=180$ (see also the right-top inset of Fig.2). This bifurcation takes place via breaking of the symmetry C of HVS (details of this bifurcation will be given elsewhere [7]). The continuation method guarantees that this branch also connects to a solution at PCF limit $(\epsilon=0)$, which corresponds to the streamwise vortex solution (SVS) previously obtained. In the homotopy parameter space spanned by $\epsilon$ and Re, the SVS is the quaternary (or higher) solution branch, while HVS is the tertiary branch in the bifurcation sequence for the laminar state. This discovery would provide us with an understanding of the vortex nature in shear flows, that would reconcile the historical controversy with respect to the distinction between the streamwise (SVS) and hairpin vortices (HVS) in turbulent shear flows.

\section{Acknowledgement}

Both of us would like to dedicate the result to our families, whose continued support enabled us to research in the field of canonical shear flows. In particular we are grateful to our fathers who over the years installed on us the enthusiasm to pursue vigorously the unknown in order to establish the truth. T.I. is grateful for the financial support received from Aston University under 
the Visiting Scholars Fund Scheme and EPSRC (GR/S70593/01). S.C.G. is grateful to a Research Invitation from the Kansai University. This work has been also supported in part by KAKENHI (19760123).

\section{References}

1. M.Nagata, J. Fluids Mech., 217, 519 (1989).

2. R. Clever and F.Busse, J. Fluids Mech., 344, 137 (1997).

3. F.Waleffe, Phys. Rev. Lett., 81, 4140 (1998).

4. F.Waleffe, Phys. Fluids, 15, 1517 (2003).

5. M.Nagata and F.Busse, J. Fluids Mech., 135, 1 (1983).

6. T. Itano and S.C. Generalis, Phys. Rev. Lett., 102, 114501 (2009).

7. T. Itano and S.C. Generalis, in preparation, (2009). 